\title{
A Distributed Transaction Method for Mitigating Three-phase Imbalance by Scheduling Electric Vehicle Charging
}

\author{
Chun Sing Lai \\ Brunel Interdisciplinary Power \\ Systems Research Centre \\ Brunel University London \\ London, UK \\ chunsing.lai@brunel.ac.uk
}

\author{
Zhaoxiong Huang \\ Department of Electrical \\ Engineering \\ Guangdong University of \\ Technology \\ Guangzhou, China \\ 3115001026@mail2.gdut.edu.cn \\ Loi Lei Lai \\ Department of Electrical \\ Engineering \\ Guangdong University of \\ Technology \\ Guangzhou, China \\ 1.l.lai@ieee.org
}

The first two authors contribute equally to the work.

Abstract-With the development of distributed trading mechanism, using Peer-to-Peer (P2P) to realize fair and developed transactions becomes a reality. $\mathrm{P} 2 \mathrm{P}$ method is used to schedule the charging behavior of electric vehicles, which can not only achieve the function of peak clipping and valley filling, but also alleviate the influence of three-phase imbalance. This paper proposes an energy transaction model which consists of two parts. The first part is a new auction in which participants can look at historical data to see how competitive they are. The second part is the interaction between the charging station operator and the participants who are not matched in the first part. Case study shows the effectiveness of this distributed trading mechanism.

Keywords-Blockchain, Energy match model, Electricity market, Three-phase imbalance

\section{NOMENCLATURE}

\section{Variables and Functions:}
$a, b$
$E p_{i}^{b}$
$b p b_{i}$
$B N_{i}^{b}$
$P$
$f$
$P e v_{i, t}^{b}$
Bidding strategy function
Possibility of EV buyers working with
Rank expectation of buyer
Evaluation price of buyer $i$
Bidding price of buyer $i$
Bidding quantity of buyer $i$
Probability function
$P e v_{i, t}^{b}$
$E^{b}$ system operator (CSO)
$F_{o b j}$

\author{
Dasheng Chen \\ Department of Electrical \\ Engineering \\ Guangdong University of \\ Technology \\ Guangzhou, China \\ 2112004023@mail2.gdut.edu.cn
}

\author{
Ahmed F. Zobaa \\ Department of Electronic and \\ Electrical Engineering \\ Brunel University London \\ London, UK \\ azobaa@ieee.org
}

$\begin{array}{cc}C_{C S O} & \text { The cost function of CSO } \\ \eta_{s a t} & \text { The social welfare function of CSO } \\ \eta_{i m b} & \text { Three-phase imbalance penalty function } \\ T A_{i, t}^{b, C S O} & \text { The transaction amount that CSO trade with the } \\ T A_{j, t}^{s, C S O} & \text { buyers } \\ m^{A}, n^{A}, m^{B}, & \text { The transaction amount that CSO trade with the } \\ n^{B}, m^{C}, n^{C} & \text { sellers }\end{array}$

Constants and Sets:

o

$b p b^{C S O}$

bps ${ }^{C S O}$

$\lambda^{G}$

$N O B$

NOS

NOT

w

$\alpha$

Breakpoint in double auction mechanism

The value of the breakpoint $a$ on the $\mathrm{x}$ axis

Price of CSO for trading with buyers

Price of CSO for trading with sellers

Cost of purchasing electricity from the grid for the CSO

Number of buyers

Number of sellers

Number of time slot

Weight value in objective function

Responsibility allocation coefficient

\section{I . INTRODUCTION}

As distributed trading matures, this approach could be applied to the scheduling of electric car charging. Because largescale electric vehicle charging can have a big negative impact 
on the grid [1]. The charging of electric vehicles is characterized by randomness and high charging power. In order to cope with the impact of the above characteristics of electric vehicle (EV) charging on the power grid, the time of use (TOU) electricity price was proposed to control the charging time of EV by referring to reference [2]. With the addition of electric buses, although the charging regularity of electric buses is obvious, it will still pose risks to the safe and stable operation of the distribution network. Reference [3] proposed a reasonable planning of quick charging stations to reduce the pressure of electric bus charging on the power grid. After the control, the impact of EV load on the power grid was reduced. Disordered charging will have a negative impact on the distribution network. In reference [4], dispatching load from the demand side can respond well to the operating pressure of distribution network. Through reasonable dispatching, the effect of peak clipping and valley filling can be realized, the uncertainty of power network can be reduced and the influence of three-phase balance can be alleviated [5]-[7]. Although the above methods can improve the operation stability of the power grid, they are all centralized management and lack of detailed models for individuals.

Peer-to-peer (P2P) may be the solution to the above problems. In reference [8], the role of blockchain technology is introduced in detail. Each individual plays a game with each other to get a state most suitable for their own interests. This method can effectively coordinate the transaction between individuals. In reference [9], P2P method is used to schedule charging of vehicle-to-vehicle ( $\mathrm{V} 2 \mathrm{~V})$, which plays the role of peak shaving and valley filling, and the charging cost of electric vehicles is reduced. There are $\mathrm{N}$ buyers and $\mathrm{M}$ sellers in a P2P trading mechanism. Auctions reflect participants' valuations, electricity consumption and other factors. Electric vehicles can be used as buyers of electric energy to participate in P2P transactions to get good charging costs. In reference [10], a vehicle-to-grid (V2G) energy trading system using blockchain was proposed. Reference [11] solved demand response by an online double auction, which is also effective in protecting participants' privacy. The above research had achieved good results, but the balance of social interests and the use of resources were not considered.

Previous work considered the feasibility between blockchain and EVs transactions and an energy transaction mechanism on the basis of blockchain was proposed [12]. Different to previous work, this paper presents an energy match model, considering the interaction between EVs transactions and load imbalance. In [13], a modified decentralized finite control set model predictive control (FCS-MPC) scheme for the distributed energy resources (DERs) was proposed to improve the power management quality of the prosumers integrated with microgrids under the condition of harmonic and unbalance loads. Three-phase unbalance is a very important factor when considering the safety and stable operation of distribution network. The main contributions of this paper are: (a) This paper proposes an energy transaction mechanism, which includes energy transaction between EVs and dispatch of the charging system operator (CSO). (b) In the dispatch of CSO, a penalty item is added to avoid too dispersed charging strategy of EVs. Moreover, the penalty term of three-phase load imbalance is added. (c) The paper linearizes the scheduling problem in the second stage.

The rest of this paper is as follows. In Section II, the energy match model is proposed concerning double auction and intelligent match. Section III describes how the model is linearized. Section IV demonstrates the validity of the model. Sections $V$ gives the conclusion and future work.

\section{PROBLEM IN MODEING}

In the previous auction research, the reuse of energy in the auction was often neglected. This section will describe how to form a double auction of energy match and an intelligent match model that takes into account the optimization of the three-phase imbalance.

\section{A. The auction}

There are two stages in an auction. In the first stage, electric vehicles can be plugged into an energy-match system on demand. In an energy-match system, buyers and sellers bid according to their own demand or supply. Buyers and sellers cannot get all the information, which is an incomplete information game. This requires the use of blockchain technology, which makes the data of transactions public. In this way, participants in the energy match system can make decisions based on current and historical data to improve their own interests.

This auction mechanism is shown in Fig.1. Buyers are ranked from highest to lowest according to demand and bidding price. On the other hand, sellers follow the opposite.

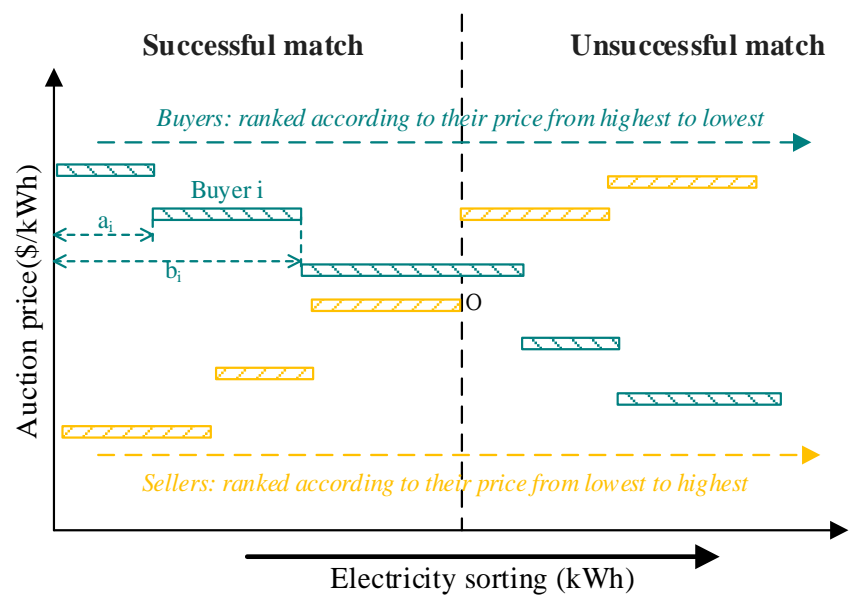

Fig.1. Schematic diagram of double auction

As shown in Fig. 1, buyer decides the bidding price according to his emergency demand for power supply. For buyers, the higher the bid price, the higher the probability of a successful match. The relationship between ranking and valuation can be expressed by the following equation.

$$
a\left(E p_{i}^{b}\right)=\sum_{k=1, k \neq i}^{N O B} P\left(b p b_{k}-b p b_{i}\right) \cdot B N_{k}^{b}-\frac{1}{2} \cdot B N_{k}^{b}
$$




$$
b\left(E p_{i}^{b}\right)=\sum_{k=1, k \neq i}^{N O B} P\left(b p b_{k}-b p b_{i}\right) \cdot B N_{k}^{b}+\frac{1}{2} \cdot B N_{k}^{b}
$$

Set the dotted line as the auction break point. The projection of dotted line $\mathrm{O}$ onto the $\mathrm{X}$-axis is equal to $\mathrm{o}$. By comparing the values $a_{i}, b_{i}$ and o, participants can estimate their auction status (success or failure). Blockchain technology can provide participants with more historical data that they can analyze to make a reasonable bid. We can obtain the buyer's optimal bidding strategy by maximizing the buyer's revenue expectation.

$b p b_{i}^{*}=\operatorname{argmax}\left\{\begin{array}{c}P\left[b\left(E p_{i}^{b}\right)<o\right] \cdot B N_{k}^{b} \cdot\left(E p_{i}^{b}-b p b_{i}\right) \\ +P\left[a\left(E p_{i}^{b}\right)<o<b\left(E p_{i}^{b}\right)\right] \\ \cdot B N_{i}^{b} \cdot\left(E p_{i}^{b}-b p b_{i}\right)\end{array}\right\}$

The seller's best bidding strategy can be obtained using the same method. Each participant submits his or her best bid as a buyer or seller.

\section{B. Smat match}

Fig. 1 shows that the transaction will be close after o point. At this time, CSO proposes a price which is better than the market clearing price, and the EV that fails to match will be willing to trade with CSO. After the deal, the central power company can store or sell the excess power to other grids.

The second stage of the auction begins after the o point, and the CSO proposes the appropriate strategy to complete the match of EVs that have not been successfully matched in the first stage.

After collecting information on participants who is willing to join the second stage, the mathematical model of CSO can be established. The objective function of CSO includes three parts, namely cost, social welfare and three-phase balance factor. The objective function is shown below.

$$
\min F_{o b j}=C_{C S O}+w_{1} \eta_{s a t}+w_{2} \eta_{i m b}
$$

The cost part is divided into two parts, namely, the cost of procuring the copy and the cost of charging and discharging as follows:

$$
\begin{aligned}
C_{C S O}= & \sum_{t=1}^{N O T}\left(\sum_{j=1}^{N O S} T A_{j, t}^{s, C S O} \cdot b p s^{C S O}-\sum_{i=1}^{N O B} T A_{i, t}^{b, C S O} \cdot b p b^{C S O}\right) \\
& +\sum_{i=1}^{N O T} \lambda^{G}\left(\sum_{i=1}^{N O B} T A_{i, t}^{b, C S O}-\sum_{j=1}^{N O S} T A_{j, t}^{s, C S O}\right)
\end{aligned}
$$

In order to make EV charging more satisfactory and try to make the charging time after CSO scheduling more concentrated, the satisfaction function is expressed as follows:

$$
\eta_{s a t}=\sum_{i=1}^{N O T}\left[\sum_{i=1}^{N O B}\left(y_{i, t}^{b}-z_{i, t}^{b}\right)+\sum_{j=1}^{N O S}\left(y_{j, t}^{s}+z_{j, t}^{s}\right)\right]
$$
below:

$$
\eta_{i m b}=\sum_{i=1}^{N O T} \sum_{X \in[A, B, C]}\left|P_{t}^{x}-\frac{1}{3}\left(P_{t}^{A}+P_{t}^{B}+P_{t}^{C}\right)\right|
$$

Where, $P_{t}^{A}=P_{t}^{A, 0}+\sum_{i=1}^{N O B} \alpha_{i}^{A} T A_{i, t}^{b, C S O}-\sum_{j=1}^{N O S} \beta_{j}^{A} T A_{j, t}^{s, C S O}(8)$

$$
\begin{aligned}
& P_{t}^{B}=P_{t}^{\mathrm{B}, 0}+\sum_{i=1}^{N O B} \alpha_{i}^{B} T A_{i, t}^{b, C S O}-\sum_{j=1}^{N O S} \beta_{j}^{B} T A_{j, t}^{s, C S O} \\
& P_{t}^{C}=P_{t}^{\mathrm{C}, 0}+\sum_{i=1}^{N O B} \alpha_{i}^{C} T A_{i, t}^{b, C S O}-\sum_{j=1}^{N O S} \beta_{j}^{C} T A_{j, t}^{s, C S O}
\end{aligned}
$$

The constraints are shown below.

(1) Constraints on which phase the EVS is assigned to charge or discharge.

$$
\begin{aligned}
& \alpha_{i}^{A}+\alpha_{i}^{B}+\alpha_{i}^{C}=\alpha_{i} \\
& \beta_{j}^{A}+\beta_{j}^{B}+\beta_{j}^{C}=\beta_{j}
\end{aligned}
$$

(2) Constraints reflecting charging and discharging times in (6) is as follows:

$$
\begin{gathered}
y_{i, t}^{b}-z_{i, t}^{b}=I_{i, t}^{b}-I_{i-1, t}^{b} \\
y_{i, t}^{b}+z_{i, t}^{b} \leq 1 \\
y_{i, t}^{b}, z_{i, t}^{b} \in[0,1]
\end{gathered}
$$

(3) Other constraints: Limit of demand balance. Constraint between dispatch variable $I_{i, t}^{b}, \alpha_{i}$ and $T A_{i, t}^{b, C S O}$.

\section{MODEL LINEARIZATION}

In Section II, there are nonlinear items in the scheduling scheme, which will affect the computational efficiency. In some cases, the absolute value can be converted to a linear form, as shown in [10].

\section{A. The linearized model for the absolute value part}

The absolute value part of Equation (8) can be linearized in the following way. To solve this problem, auxiliary variables $m^{A}, n^{A}, m^{B}, n^{B}, m^{C}, n^{C}$ are introduced.

Equation (8) can be rewritten as follows:

$$
\sum_{t=1}^{N O T} \sum_{X \in[A, B, C]}\left(m_{t}^{X}+n_{t}^{X}\right)
$$

s.t.

$$
\begin{aligned}
& P_{t}^{A}-\frac{P_{t}^{A}+P_{t}^{B}+P_{t}^{C}}{3}=m_{t}^{A}-n_{t}^{A} \\
& P_{t}^{B}-\frac{P_{t}^{A}+P_{t}^{B}+P_{t}^{C}}{3}=m_{t}^{B}-n_{t}^{B} \\
& P_{t}^{C}-\frac{P_{t}^{A}+P_{t}^{B}+P_{t}^{C}}{3}=m_{t}^{C}-n_{t}^{C} \\
& m_{t}^{X}, n_{t}^{X} \geq 0 \quad X \in[A, B, C]
\end{aligned}
$$

Other constraint $s$ 
This article has been accepted for publication in a future issue of this conference proceedings, but has not been fully edited. Content may change prior to final publication. Citation information: DOI10.1109/ACPEE51499.2021.9437066, Proceedings of the 20216 th Asia Conference on Power and Electrical Engineering, ACPEE 2021

(14)

B. The linearized model of the product of variables

Equation (14) can be replaced as follows:

$$
P_{t}^{A}=P_{t}^{A, O}+\sum_{i=1}^{N O B} \Delta P_{i, t}^{A, b}-\sum_{j=1}^{N O S} \Delta P_{j, t}^{A, S}
$$

With constraints and $\mathrm{M}$ is a big enough number.

$$
\begin{gathered}
-\left(1-\alpha_{i}^{A}\right) \cdot M \leq \Delta P_{i, t}^{A, b}-W_{i, t}^{b, C S O} \leq\left(1-\alpha_{i}^{A}\right) \cdot M \\
0 \leq \Delta P_{i, t}^{A, b} \leq \alpha_{i}^{A} \cdot M
\end{gathered}
$$

\section{CASE STUDY}

In the above trading mechanism, EVs explore auction information more deeply, improving the probability of successful transactions and pursues higher profits. CSO not only considers the demand/supply match problem of EVs, but also aims to alleviate the load imbalance of nearby users. This case adopts EVs behavior data and three-phase load as in [11] and [12] respectively, and EVs data includes the charging/discharging amount and time of 150 buyers and 120 sellers.

\section{A. The transaction results of participants}

In this part, we will compare the experimental results whether EVs participate or not in the proposed trading mechanism. Table 1 shows the transaction results of EVs when they participate this transaction or not.

\begin{tabular}{|c|c|c|}
\hline Participant & Buyer & Seller \\
\hline $\begin{array}{l}\text { Total cost/benefit in } \\
\text { double auction (\$) }\end{array}$ & 103.63 & 103.63 \\
\hline $\begin{array}{l}\text { Total cost/benefit in } \\
\text { smart match }(\$)\end{array}$ & 37.65 & 101.36 \\
\hline $\begin{array}{l}\text { Total cost/benefit in } \\
\text { energy match process (\$) }\end{array}$ & 271.73 & 206.16 \\
\hline $\begin{array}{c}\text { Total cost/benefit } \\
\text { without participation (\$) }\end{array}$ & 284.11 & 188.24 \\
\hline
\end{tabular}

Table 1: Cost (Revenue) of participants whether to participate or not in the proposed transaction mechanism

From the comparison of the experimental results, we can conclude that participating in this transaction mechanism is profitable for the participants. The average buyer's cost declines from $\$ 1.89$ to $\$ 1.81$, and the average seller's benefit rises from $\$ 1.57$ to $\$ 1.72$, which greatly encourages EVs to participate in this mechanism.

\section{$B$. The influence on three-phase load}

Another important point is that the electricity traded from the EVs will be transferred to nearby buildings to alleviate the threephase load imbalance. Fig. 2 shows the three-phase load of nearby building, and Fig. 3 shows the three-phase load after smart match.

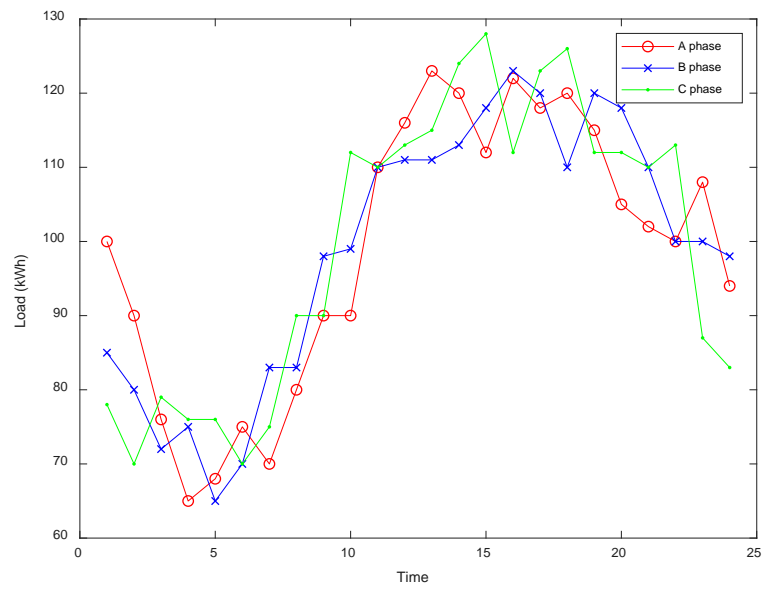

Fig.2. Three-phase load of nearby building

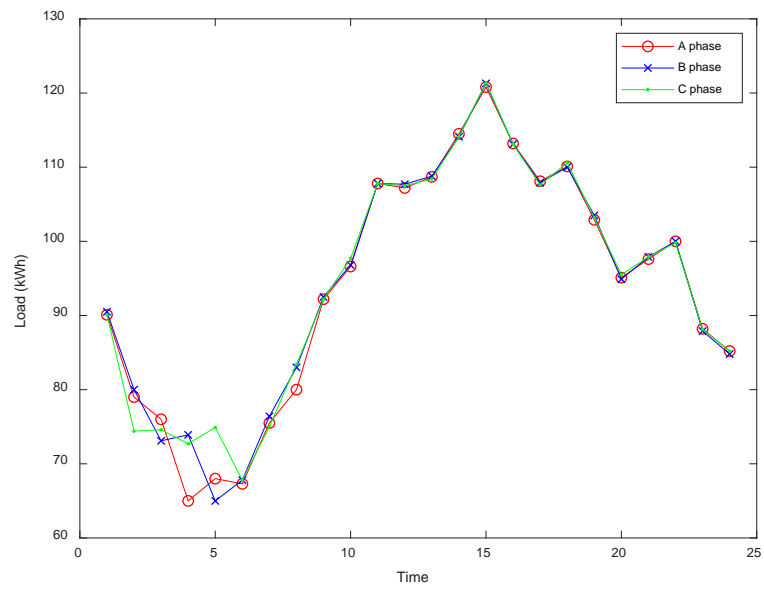

Fig. 3. Three-phase load after smart match

From Figs. 2 and 3, it can be sent that smart match is effective for the elimination of load imbalance. $\eta_{\text {imb }}$, which reflects the three-phase imbalance, is as high as $319.33 \mathrm{kWh}$ in Fig. 2, and this number is reduced to $47.2 \mathrm{kWh}$ in Fig. 3. Therefore, it is feasible to take EVs energy transaction into account to eliminate load imbalance.

\section{CONCLUSION}

A new energy match mechanism is proposed in this paper for mitigating three-phase imbalance by scheduling electric vehicle charging. The mechanism iincludes two-way auction and smart match. This paper presents a new incomplete information game in two-way auction. The approach proposed in this paper is more effective when more transparent historical information is available. Using blockchain technology, participants have access to open historical data. The main benefit for the buyer was the reduction in the buyer's cost from $\$ 1.8941$ to $\$ 1.5084$. Different from the traditional auction mechanism, this auction mechanism increases the intelligent match, which is useful to the energy recovery and utilization of electric vehicles. From the point of view of power grid, this energy management mechanism can play the role of peak clipping and valley filling. 
This article has been accepted for publication in a future issue of this conference proceedings, but has not been fully edited. Content may change prior to final publication. Citation information: DOI10.1109/ACPEE51499.2021.9437066, Proceedings of the 2021 th Asia Conference on Power and Electrical Engineering, ACPEE 2021

Moreover, the objective function considers the optimization of three-phase imbalance of the power grid. The system using this method reduces the three phase imbalance from the original $319.33 \mathrm{kWh}$ to $47.2 \mathrm{kWh}$.

Future work can take into account the voltage offset of the grid nodes. Electric vehicles can be used as a mobile energy storage system for reasonable dispatching to achieve economic and stable power grid operation.

\section{ACKNOWLEDGEMENT}

The authors are grateful to the conference committee and Ms Amber Cao, Conference Secretary of ACPEE 2021 for the paper invitation.

This work is sponsored by Brunel University London BRIEF Funding; the Department of Finance and Education of Guangdong Province 2016 [202]: Key Discipline Construction Program, China; Education Department of Guangdong Province: New and Integrated Energy System Theory and Technology Research Group [Project Number 2016KCXTD022]. We are grateful for support from the DTE Network+ funded by EPSRC grant reference EP/S032053/1.

\section{REFERENCES}

[1] Küfeoğlu S. and Pollitt M. G.: 'The impact of PVs and EVs on domestic electricity network charges: A case study from Great Britain,' Energy Policy, vol. 127, pp. 412-424, Apr. 2019, doi: 10.1016/j.enpol.2018.12.012.

[2] Feng, K., Zhong, Y., Hong, B., Wu, X., Lai, C.S. and Bai, C., 'The impact of plug-in electric vehicles on distribution network,' 2020 IEEE International Smart Cities Conference (ISC2) (pp. 1-7). IEEE.

[3] Wu, X., Feng, Q., Bai, C., Lai, C.S., Jia, Y. and Lai, L.L., 'A novel fastcharging stations locational planning model for electric bus transit system,' Energy, 2021, p.120106.

[4] Davarzani S., Pisica I., Taylor G. A., and Munisami K. J., 'Residential demand response strategies and applications in active distribution network management,' Renewable and Sustainable Energy Reviews, vol. 138. Elsevier Ltd, Mar. 01, 2021, doi: 10.1016/j.rser.2020.110567.

[5] Dang, Q., 'Electric vehicle (EV) charging management and relieve impacts in grids,' 2018 9th IEEE International ymposium on Power Electronics for Distributed Generation Systems (PEDG), Charlotte, NC, 2018, pp. 1-5.

[6] Yamasaki, J., Tominaga, R., Ishii, Y., Shimizu, A., Kinoshita, S., and Wakao, S.: 'Optimization of installation and operation for retail store with photovoltaic, storage battery and EV quick charger,' 2011 37th IEEE Photovoltaic Specialists Conference, Seattle, WA, 2011, pp. 001893001896.

[7] Su, X., Li, W., and Li, X., 'Research on ordered charging of battery swapping station based on adaptive genetic algorithm,' 2019 IEEE Sustainable Power and Energy Conference (iSPEC), Beijing, China, 2019, pp. 792-796.

[8] Lai, C.S., Lai, L.L., and Lai, Q.H., Blockchain Applications in Microgrid Clusters. In Smart Grids and Big Data Analytics for Smart Cities (pp. 265-305). , 2020, Springer, Cham.

[9] Huang, Z., Chen, D., Lai, C.S., Zhao, Z., Lai, L.L., and Wang, M., 'A distributed transaction mechanism for electricity market with electric vehicles and blockchain,' 2020 8th International Conference on Power Electronics Systems and Applications (PESA), Dec 2020, (pp. 1-5). IEEE.

[10] Hong J., Oh E., Yang Y., Roh K., Oh M. and Kim J., 'Consortium blockchain-based V2G energy trading system using tokens,' International Conference on Information and Communication Technology
Convergence (ICTC), Jeju, Korea (South), 2020, pp. 677-682, doi: 10.1109/ICTC49870.2020.9289330.

[11] Li, D., Yang, Q., Yu, W., An, D., Zhang, Y., and Zhao, W., 'Towards differential privacy-based online double auction for smart grid,' IEEE Transactions on Information Forensics and Security, 15, 2020, pp. 971986.

[12] Yin, C., Wang, F., Ding, X. , Tian, C., and Duan, P., 'A novel compensator for three-phase load unbalance of the low voltage distribution network,' 2017 Chinese Automation Congress (CAC), Jinan, China, 2017, pp. 7518-7521, doi: 10.1109/CAC.2017.8244138.

[13] Zhao, Z., Zhang, J., Yan, B., Cheng, R., Lai, C.S., Huang, L., Guan, Q., and Lai, L.L., 'Decentralized finite control set model predictive control strategy of microgrids for unbalanced and harmonic power management,' IEEE Access, 8, 2020, pp.202298-202311.

[14] Huang, Z., Li, Z., Lai, C.S., Zhao, Z., Wu, X., Li, X., Tong, N., Lai, L.L, 'A novel power market mechanism based on blockchain for electric vehicle charging stations,' Electronics 2021, 10, 307

[15] Kikusato, H., Fujimoto, Y., Hanada, S., Isogawa, D., Yoshizawa, S., Ohashi, H., and Hayashi, Y., 'Electric vehicle charging management using auction mechanism for reducing PV curtailment in distribution systems,' IEEE Transactions on Sustainable Energy, 11, (3), July 2020, pp. 13941403.

[16] Muratori M., 'Impact of uncoordinated plug-in electric vehicle charging on residential power demand,' Nature Energy, 3, 2018, pp. 193-201.

[17] Shi B.S., Zhang J., He Y.,Na Ding N., and Qu Z.Y., 'Electricity consumption behavior of single-phase power consumers in distribution network based on dynamic game theory,' Automation of Electric Power Systems, 2017, 87-91+139. doi:CNKI:SUN:DLXT.0.2017-14-012. 